\title{
EMPRESAS MULTINACIONALES Y SUS EFECTOS EN LOS PAÍSES MENOS DESARROLLADOS*
}

\author{
Mauricio Lascurain Fernández*
}

\section{RESUMEN}

En el presente artículo se da a conocer la dinámica de negociación entre una empresa multinacional y el gobierno de un país menos desarrollado, así como sus efectos en el país de acogida.

Palabras clave: inversión extranjera directa, negociación, globalización económica, países menos desarrollados, enfoques teóricos.

Clasificación JEL: F23, F51, F02, O10, B49.

\section{ABSTRACT}

The aim of this paper is to identify the bargaining dynamics between a multinational firm and the government of a less developed country, and the effects of this firm in the country.

Key words: foreign direct investment, bargaining strategies, economic globalization, less developed countries, theoretical approaches.

Classification JEL: F23, F51, F02, O10, B49.

- Fecha de recepción: 01/09/2011. Fecha de aceptación final: 07/02/2012.

* El Colegio de Veracruz. mlascurain@colver.edu.mx 


\section{INTRODUCCIÓN}

De acuerdo con la UNCTAD (2008), los países menos desarrollados (PMD) o adelantados son aquellos que muestran los indicadores socioeconómicos más bajos, especialmente en el índice de desarrollo humano (IDH). ${ }^{1}$ Actualmente la Organización de las Naciones Unidas considera 48 países en esta categoría, los cuales se encuentran especialmente en África, Asia, Oceanía y un país del Caribe. Las principales características de dichas naciones son su vulnerabilidad económica, bajo desarrollo humano y la falta de instituciones fuertes y confiables. Al tener pocas perspectivas de crecimiento por sus propias estructuras socioeconómicas, los PMD ven una oportunidad de desarrollo con la llegada de empresas multinacionales (EMN) a su territorio.

Sin embargo, las relaciones entre las empresas multinacionales y los PMD han sido conflictivas a lo largo del tiempo; no obstante, éstos tienden a disminuir por dos factores esenciales: a) el aceleramiento del proceso de globalización económica en los últimos 30 años, y b) los PMD han observado que hay ciertas ventajas para impulsar el crecimiento económico al momento en que se instalan las EMN en su territorio. La gran preocupación de los gobiernos nacionales en los países menos desarrollados es que algunas EMN tienen un poder de influencia y negociación mayor que los propios gobiernos, lo que pone en entredicho una posición favorable de negociación para los PMD. La gran mayoría de esas empresas cuentan con nuevas tecnologías, están dispuestas a invertir en el PMD y desarrollan las habilidades de la población, entre otros factores, lo que las pone en una ventaja inicial en la dinámica de negociación.

El objetivo de este artículo es conocer precisamente esa dinámica de negociación, así como sus efectos en los PMD. El trabajo se ha dividido en cuatro secciones. En la primera se hace un acercamiento al concepto de EMN. En la segunda se abordan las principales teorías de esas empresas, a fin de comprender las

${ }^{1}$ Además, la UNCTAD (2008) considera que un país menos desarrollado debe reunir los siguientes criterios: a) baja renta per cápita: tres años de media de 750 dólares para la inclusión de países en la lista y de 900 dólares para la exclusión; b) debilidad en recursos humanos: comprobada con los indicadores de nutrición, salud, educación y alfabetización de adultos; c) vulnerabilidad económica: producida por la inestabilidad de la producción agraria, de las exportaciones de bienes y servicios, la concentración en la exportación de ciertas mercancías (monocultivos), la escasa importancia económica de las actividades no tradicionales (economía de subsistencia), las dificultades consiguientes a la pequeña escala del mercado nacional y las empresas o la repetición de desastres naturales, guerras o conflictos internos que causen desplazamientos masivos de la población. 
diferentes interpretaciones sobre el fenómeno. En la tercera se profundiza sobre los efectos de las multinacionales en los PMD. La cuarta sección esquematiza las estrategias de negociación de los PMD frente a las EMN. Finalmente, con los datos arrojados por la investigación, se realizarán algunas conclusiones generales.

\section{CONCEPTOS BÁSICOS DE LAS EMPRESAS MULTINACIONALES}

El actual proceso de globalización económica no se entiende sin la apertura comercial, las innovaciones tecnológicas y sobre todo sin la liberalización de las regulaciones nacionales sobre inversión extranjera que se ha hecho en los últimos años. Como parte de estos avances, las EMN han desempeñado un papel fundamental en la expansión de la integración económica internacional, no sólo por el volumen que generan y los flujos financieros que producen, sino también porque han contribuido a modificar los patrones comerciales de un comercio interindustrial hacia uno intraindustrial.

Las empresas multinacionales han sido conceptualizadas de diferente manera; algunos autores se refieren a ellas como empresas globales, internacionales o trasnacionales (ETN). Sin embargo, Hisrt y Thomposn (1996) sugieren que estas empresas aún no son lo suficientemente globales en su funcionamiento y operación como para ser verdaderamente trasnacionales; por lo tanto, a lo largo de este trabajo nos referiremos a ellas como empresas multinacionales.

Es posible encontrar varias definiciones de lo que es una EMN; por ejemplo, Willetts (2001: 362) la define como "una compañía con base en un país, la cual tiene filiales que mantienen tratos comerciales con una sociedad o gobierno en otro país". Durán (2001: 95) considera que una EMN es aquella "que posee (coordina y controla) filiales en uno o más países extranjeros". Evans y Newnham (1998) proponen que la EMN es una organización creadora de ganancias que controla bienes al menos en dos países. Para Gilpin (2001: 278) es una "compañía de una nacionalidad en particular, la cual es propietaria de forma parcial o total de filiales dentro de otra economía nacional". ${ }^{2}$ Como podemos observar, en las definiciones anteriores hay un común denominador, que es el control de una actividad empresarial en el exterior y presencia en al menos dos países, que pueden ser identificados como el país de origen (home state), que es aquel al que pertenece la empresa, y el país de acogida (host state), que es aquel en que la empresa es dueña de bienes o tiene filiales.

${ }^{2}$ Para efectos de este trabajo, nos basaremos en esta última definición. 
A pesar de que en las ciencias sociales es complicado establecer el grado de causalidad entre las variables, algunos expertos (Sanna-Randaccio y Veugelers, 2003; Barrios et al, 2003; Feldstein, 2000; Gilpin, 2001; Bhagwati, 2004; Wolf, 2005; Sala-i-Martin, 2006) cosideran la IED como un catalizador del crecimiento económico, cuyos principales beneficios se dan mediante la transferencia de tecnología, en especial en forma de nuevas variedades de insumos de capital, lo que no se logra con inversiones financieras ni con el comercio de bienes y servicios, y contribuye a una mayor integración del país al comercio internacional. También se ha comprobado que promueve la competencia en el mercado nacional de insumos y es capaz de fomentar la creación de un ambiente empresarial competitivo al incrementar el desarrollo de los negocios. Asimismo, los países que reciben IED normalmente obtienen capacitación para sus empleados en el desempeño de las nuevas tareas, lo que contribuye al desarrollo del capital humano de esa zona específica. Además, la derrama económica que produce la IED incrementa la recaudación local para los respectivos niveles de gobierno.

No obstante, su presencia y efectos en las economías nacionales son también punto de partida de críticas, como por ejemplo que las EMN empobrecen al país receptor y explotan a los trabajadores nacionales. Otro aspecto que se reprocha es que son más poderosas que los países pequeños y que dañan la soberanía nacional (Anderson y Cavanagh, 2000). Estas situaciones las analizaremos más adelante.

En términos generales, la IED tiene el propósito de acaparar parcial o totalmente el mercado o la producción en una economía de otro país; esta inversión se puede dar en servicios, manufactura o en la extracción y explotación de recursos naturales. Estas acciones se realizan en actividades ya existentes o creando nueva inversión; a esto se le ha denominado como greenfield (Gilpin, 2001; Durán, 2001; Dunning y Narula, 1997). A estas inversiones en el extranjero se les dota de personalidad jurídica y en sí mismas o conjuntamente con otras constituyen unidades empresariales residentes en el país de acogida. Desde la perspectiva de este último se contabilizan como empresas, y desde la del inversionista son filiales en el exterior. Para obtener el control de una empresa en otro país, la Organización de las Naciones Unidas (ONU) propone que no es necesaria la participación total y mayoritaria en el capital social de una empresa. La misma organización engloba en el término de filiales tanto a las subsidiarias (la compañía matriz posee más de $25 \%$ de las acciones con derecho a voto), como a las afiliadas (entre 10 y $25 \%$ de las acciones) (Durán, 2001).

Históricamente, la actividad de la EMN en el sector primario ha sido primordial para el desarrollo de éste. El crecimiento económico a lo largo de los siglos XV al XX obligó a las empresas a buscar recursos naturales en otros continentes, 
especialmente en los PMD, siendo el petróleo, aluminio y cobre, entre otros, los principales recursos explotados. No es casualidad que las EMN hayan sido criticadas por los sectores extractivos de los países de acogida como empresas altamente explotadoras. La repercusión económica que tuvieron en los mercados locales por lo general fue mínima, comparada con las ganancias obtenidas por la extracción y venta de hidrocarburos o aquel recurso natural explotado (Taylor, 1999; Lipsey, 2001). En el caso de México, en la década de 1930 se tomaron medidas extremas al expropiar el sector petrolero que estaba en manos de EMN extranjeras, las cuales obtenían grandes ganancias a costa de unas condiciones de trabajo deplorables. ${ }^{3}$

Respecto a las manufacturas o sector secundario, éste ha sido tradicionalmente el más importante de la actividad de las EMN. El aumento de las manufacturas, principalmente en el siglo XX, incrementó la importancia de los países desarrollados (PD), como receptores de IED, destinándose gran parte de ella a esos países. En los PMD la inversión de manufactura ha sido muy selectiva en su localización, prefiriendo instalarse en países donde hay un mayor desarrollo, como es el caso de los NIC. ${ }^{4}$ En este sector, el principal elemento de intercambio ha sido la transferencia de tecnología, la cual está controlada por las EMN por medio de patentes y se calcula que aproximadamente $70 \%$ de los pagos por derechos o tecnología que se realizan en el mundo se hacen entre las multinacionales y sus filiales (De la Dehesa, 2004). Sin embargo, el efecto de esta transferencia de tecnología no ha podido ser aprovechada de manera óptima en muchos PMD. Por ejemplo, si el sistema de producción de una filial de la EMN necesita mano de obra calificada, dicho sistema no será apropiado en donde la mano de obra es barata y no calificada. Esto limita aún más la posibilidad de que los PMD entren en las redes globales de las EMN (Taylor, 1999).

La localización de la actividad manufacturera no ha sido tan limitada geográficamente como la extractiva. En el momento de decidir en dónde se va a localizar una subsidiaria de la EMN, ésta puede escoger entre diferentes destinos. Además, si de antemano hay un interés del país de acogida por atraer esa inversión, la EMN ofrecerá indicios positivos de que querrá entrar a ese mercado, en muchos casos apoyada por su país de origen (Lipsey, 2001). Naturalmente, estas facilidades incrementarán el poder de negociación de la empresa en el pacto final; por ejemplo, se le puede ofrecer un trato preferencial con los sindicatos, reducción de impuestos, etc. En términos generales, la actividad manufacturera de una EMN suele ser más integrada e influyente en la vida económica y social del país de acogida.

${ }^{3}$ Para profundizar en el tema, se puede consultar la página web del Instituto Mexicano del Petróleo (IMP).

${ }^{4}$ Por sus siglas en inglés newly industrialized countries. 
En el caso del sector servicios, las EMN desempeñan un papel importante para su propagación por el mundo; por un lado, los bancos, seguros y demás servicios financieros y, por otro, el turismo. Al igual que el sector manufacturero, las multinacionales de este sector suelen instalarse preferentemente en PD y en un selecto número de PMD, complicando una vez más la integración de éstos a las redes globales. Este sector ha estado estrechamente ligado al sector secundario, ya que la movilización internacional de la industria de servicios por lo general sigue a la industria manufacturera. Gracias a esto, en los últimos años la IED de la industria de servicios es el sector de las EMN que crece más rápido (Goldberg, 2004).

En la actualidad las EMN, sea cual sea el sector económico en el que se desarrollen, son responsables de dos tercios de las exportaciones mundiales de bienes y servicios y representan cerca de $10 \%$ de todas las ventas locales mundiales (De la Dehesa, 2004), lo que refleja que son agentes no gubernamentales muy importantes en la expansión de la actividad económica mundial. Sin embargo, hay diferentes interpretaciones teóricas sobre las motivaciones de inversión de las EMN. A continuación analizaremos algunas de las más importantes.

\section{TEORÍA DE LAS EMPRESAS MULTINACIONALES}

La empresa multinacional es un fenómeno complejo desde un punto de vista conceptual, que ha sido analizado desde diferentes perspectivas. Esto ha producido una extensa literatura sobre el tema, que sin duda enriquece el debate científico. Sin embargo, un buen punto de partida es el expuesto por Gilpin (2001), quien en términos generales propone que este fenómeno puede ser abordado desde el punto de vista neoclásico, pasando por la interpretación empresarial, hasta la propuesta de la economía política. En el caso de los economistas neoclásicos, éstos consideran que el mercado es el principal actor en una economía, minimizando de esta manera la participación de las instituciones como agentes influyentes. Desde esta perspectiva, el comportamiento de las empresas estará determinado principalmente por el mercado, por lo que la nacionalidad de las empresas y su forma de operación, ya sea local o internacional, tienen poca relevancia para explicar su conducta (Gilpin, 2001: 279). Krugman y Obstfeld (1999) consideran que la teoría moderna de las EMN trata de contestar dos preguntas fundamentales: ¿por qué producir en varios países en lugar de en uno solo? y ¿por qué su producción en diferentes localizaciones se realiza mediante la misma empresa y no por empresas separadas? La primera pregunta se refiere a la teoría de la localización, mientras que la segunda hace referencia a la teoría de la internalización. 
La primera teoría se refiere a que la localización de la producción estará determinada en gran medida por los recursos necesitados. Por ejemplo, los fabricantes de PC o portátiles localizan sus recursos de diseño intensivo en tecnología en el Silicon Valley al este de Estados Unidos y sus plantas de ensamblaje intensivas en mano de obra estarán en donde ésta sea más barata. Esta teoría, a su vez, plantea otras alternativas de localización, como son los costos de transporte y las barreras al comercio. En general, la teoría de la localización plantea que las decisiones de una EMN en lo concerniente a dónde producir, se basan en los principios de la teoría general de comercio internacional (Krugman y Obstfeld, 1999).

Respecto a la teoría de internalización, ésta propone que las operaciones con países diferentes conllevan a elevados costos de transacción, los cuales pueden reducirse internalizándolos, y de esta manera se favorece la creación de las EMN. Krugman y Obstfeld (1999) coinciden en que permanentemente hay transacciones entre las filiales de una EMN alrededor del mundo en donde el output de una filial por lo general es el input de la producción de otra, mientras que la tecnología desarrollada en una filial puede utilizarse en otras. Actividades como éstas son las que mantienen unida a la EMN y probablemente la empresa existe para facilitar esas transacciones. Sin embargo, esto no significa que todas las transacciones deban realizarse dentro de la empresa, pues en la mayoría de los casos los componentes se pueden vender en un mercado y la tecnología puede venderse a otras empresas. Lo anterior significa que estas transacciones suelen ser más baratas cuando se realizan dentro de la empresa y a su vez se puede sacar provecho por su comercialización.

No obstante, la postura de Krugman y Obstfeld no alcanza para explicar el papel que desempeñan las EMN en el sistema económico mundial, ya que consideran que éstas no son un factor determinante en la economía internacional. Asimismo, argumentan que la IED y otros resultados económicos producidos por las mencionadas empresas tienen pocos efectos sobre la distribución económica global y no podrían distinguirse de aquellos del comercio internacional. Para Krugman y Obstfeld el principal efecto de la IED se encuentra en el ámbito nacional, ya que ocasiona cambios en la distribución del ingreso y no tanto en escala internacional (Gilpin, 2001).

La segunda perspectiva sobre el comportamiento de las EMN se puede abordar desde la perspectiva empresarial, como Raymond Vernon (1973), John Dunning (1999), Michael Porter (1990), entre otros. Las ideas principales de esta postura se centran en diversos estudios de las operaciones empresariales en escala internacional que tienen la finalidad de conocer los beneficios de la IED tanto en el país de origen como en el de acogida. 
La aportación de Vernon (1973) se basa principalmente en su teoría del "ciclo de vida del producto" que propone que la ubicación de la producción de ciertos tipos de productos cambia a medida que atraviesan por sus ciclos de vida, los cuales consisten en cuatro etapas: introducción, crecimiento, madurez y declinación. Para Vernon las empresas desarrollan productos porque hay una necesidad y un mercado inmediatos para ellos. Por ejemplo, una empresa estadounidense es más apropiada para desarrollar un producto para su mercado o una francesa para el mercado suyo. Además, desde esta perspectiva gran parte de la nueva tecnología se genera en los PD debido a cierta combinación de factores, como la competencia entre las empresas, la demanda de los consumidores, la disponibilidad de científicos e ingenieros, y altos ingenieros.

A medida que se avanza en el ciclo de vida del producto, las EMN empiezan a desplazarse a otros países, en donde el último que produce el producto (fase de declinación) se encuentra en un PMD. Cuando llega la producción a ellos, los procesos se han vuelto más estandarizados, el producto ya no es intensivo en capital y por lo general los PMD exportarán a los mercados declinantes o nichos reducidos en los PD. A pesar de que la teoría de Vernon explica cómo las EMN se van expandiendo por el mundo de acuerdo con el ciclo de vida de sus productos, ésta tiene ciertas limitaciones. Por ejemplo, sólo se puede confirmar este tipo de teoría en algunos productos con ciclos de vida extremadamente cortos, como los de consumo no perecederos, los materiales sintéticos y los aparatos electrónicos, los cuales están expuestos a la obsolescencia en su uso y fabricación, que al final su producción internacional es limitada. Esta teoría trata de explicar el proceso y no tanto las razones por las cuales las EMN deciden movilizarse en todo del mundo.

Una segunda propuesta de la perspectiva empresarial es la teoría ecléctica de Dunning (1999), la cual destaca que la tecnología ha sido un factor esencial para el desarrollo de las EMN. En este sentido, las comunicaciones y el transporte han permitido que éstas puedan organizar y administrar sus servicios y producción en escala global. De acuerdo con Dunning (1999), el enfoque ecléctico está fundamentado en tres teorías económicas: la primera es la de la organización industrial, la cual explica cómo es posible que un grupo de empresas pueda adquirir y conservar una ventaja competitiva o conjunto de ellas frente a otro grupo de empresas. La segunda es la teoría de la empresa, la cual trata de explicar la forma organizacional por la cual la empresa crea, aumenta y usa unas ventajas competitivas. Finalmente, está la teoría de localización, explicada anteriormente.

$\mathrm{El}$ análisis de las tres teorías anteriores han permitido crear un nuevo paradigma llamado OLI, el cual Dunning (1999: 8) define como "en cualquier momento del tiempo, la propiedad y los patrones de actividad de las EMN dependerán, i) de la 
configuración de sus ventajas competitivas (ownership [O] specific) vis-à-vis aquellas empresas no multinacionales; ii) de la atracción de competitividad de un país o región (location [L] specific) vis-à-vis otros países, y iii) de los beneficios de las empresas por explotar estas dos ventajas internalizando el mercado para las ventajas de O specific, dando como resultado la ventaja de internalización (I)". De esta manera, las EMN logran tener más éxito económico que las empresas nacionales.

La tercera propuesta de la perspectiva empresarial es la teoría de la estrategia de Michael Porter (1990), la cual propone que los negocios internacionales o EMN se caracterizan por una cadena de valor que va desde la extracción, pasando por la producción, hasta la mercadotecnia. De esta manera, cada una de las empresas deberá decidir en cuál y en cuántas actividades incursionar y en dónde se instalará. Por tanto, la decisión dependerá de la estrategia competitiva de cada EMN.

En general, las propuestas desde la perspectiva empresarial se enfocan hacia adentro de la empresa, es decir, consideran que el crecimiento de las EMN se debe a una estrategia empresarial y organizacional en la que el motivo principal de su división internacional en la producción se debe, fundamentalmente, a la búsqueda de la disminución de los costos de ésta. Sin embargo, dejan de lado aspectos fundamentales como los fenómenos internacionales, los cuales son los que aborda la perspectiva de la economía política, que a continuación se desarrolla.

Al menos se tienen dos posturas de la economía política que explican el comportamiento de las EMN: los marxistas y la interpretación State-Centric. Uno de los principales promotores de las ideas marxistas sobre las EMN es Hymer (1976), quien propone dos leyes esenciales del monopolio del capitalismo. La primera se refiere al aumento del tamaño de la empresa, la cual crece tanto a los ámbitos nacionales como internacionales, creando una especie de producción centroperiferia, en la que el centro está representado por los PD y en la periferia por los PMD. La segunda ley propone que las EMN generan un desarrollo desigual, ya que explotan a la periferia para obtener el máximo beneficio para ellos. Por lo tanto, de acuerdo con la interpretación marxista, la característica principal de las EMN es que son agentes explotadores de los PMD.

El enfoque State-Centric propone que el incremento de las EMN no hubiera sido posible sin un ambiente político-económico internacional favorable, el cual "fue permisible gracias al esfuerzo de un poder dominante, cuyos intereses económicos y de seguridad favorecieron una apertura comercial internacional" (Gilpin, 2001: 288). Este poder dominante se refiere a Estados Unidos, que a finales de la segunda guerra mundial surge como la hegemonía mundial, creando así el ambiente propicio para la expansión de sus empresas alrededor del mundo. De esta manera, el enfoque de la política económica nos permite observar que las 
EMN se encuentran inmersas en entornos particulares de los países en los que esté presente, así como en el ámbito regional e internacional en general.

El análisis de las teorías anteriores nos permite observar que la atracción de la IED hacia un mercado específico se encuentra condicionada por factores de distinta índole, entre los que resaltan los siguientes. ${ }^{5}$

Factores macroeconómicos. Reflejan la estabilidad, competitividad y confiabilidad del país, la región o microrregión para emprender negocios. Para ello, las EMN toman como medidas la tasa de crecimiento, la inflación actual e histórica, el producto interno bruto (PIB), la relación ahorro-inversión, el saldo en la cuenta corriente como proporción del PIB, el tipo de cambio, la deuda externa, el dinamismo del comercio exterior y el índice de riesgo país, entre otros factores.

Mercado interno. El potencial del mercado en términos de demanda es un elemento que las EMN toman en cuenta al momento de decidir su ingreso en un determinado país o región. En este sentido, los factores que se analizan son el tamaño de la población, la proximidad geográfica con otros mercados externos, el PIB percápita, el crecimiento de la población, la distribución de la riqueza, la facilidad de realizar negocio, el ahorro y el potencial de consumo. Además se toman en cuenta las industrias conexas o de apoyo, así como la estrategia, estructura y rivalidad de las empresas ya consolidadas en el mercado.

Factores de producción. Un tercer elemento que toma en cuenta la IED al momento de localizar un nuevo mercado es la calidad de los factores de producción del país o la región de acogida. Entre estos factores se encuentra la calidad de la mano de obra, la disponibilidad de capital y el grado de desarrollo de la infraestructura. Referente a la mano de obra, ésta es de gran importancia para la EMN dependiendo de la complejidad de la cadena de valor del producto o servicio que se preste, por lo que puede ser una mano de obra ya sea altamente calificada o bien con menos preparación. Asimismo, las EMN prefieren instalarse en regiones donde haya una amplia disponibilidad de capital que se traduce en créditos para proyectos productivos, bajos requisitos y garantías para acceder a ellos y bajas tasas de interés, entre otros.

Por su parte, la infraestructura en la región es un factor que puede facilitar $\mathrm{o}$, en su defecto, dificultar las actividades de esas empresas, por lo que el grado de desarrollo de aquélla puede ser fundamental para la instalación de las IED. Por lo tanto, es posible afirmar que las EMN basan su inclusión en un mercado en los principios de la teoría de localización, la cual plantea que las decisiones de una EMN en lo concerniente a dónde producir estará determinada por los recursos necesitados (Krugman y Obstfeld, 1999).

${ }^{5}$ Para un análisis más profundo sobre los factores de atracción de la IED a un país, véase UNCTAD (2007 y 2008a) y Bhagwati (2004). 
Políticas públicas. La mayoría de las autoridades competentes en los diferentes niveles de gobierno instrumentan políticas públicas destinadas a atraer y promover la IED, como la disminución de impuestos, la disponibilidad de asentamientos, el número de parques industriales, etcétera.

Estabilidad institucional. Dentro de este factor se encuentra la eficiencia gubernamental en cuanto a los servicios públicos ofrecidos, el nivel y la calidad del aparato burocrático y de corrupción del país, la entidad federativa o el municipio. Además, se incluye en este apartado el grado de seguridad jurídica que puedan ofrecer los diferentes niveles de gobierno a las multinacionales, ya sea en estabilidad política, laboral, respeto a los derechos de propiedad y, en general, un efectivo Estado de derecho. Sin embargo, ¿cuáles son los principales efectos de las EMN en el país de acogida? A continuación analizaremos las principales consecuencias de ese tipo de empresa en el país huésped.

\section{EFECTOS DE LAS EMPRESAS MULTINACIONALES EN EL PAÍS DE ACOGIDA}

Las relaciones entre las EMN y los gobiernos de acogida, en especial en los PMD, son complejas. Esto se debe principalmente a que aquéllas buscan la maximización de sus beneficios económicos, mientras que los gobiernos de dichos países buscan la maximización del valor agregado que les pueda dejar la actividad de la multinacional, en especial aquella que se queda dentro de las fronteras del país. Asímismo, debido a sus características de aportación de tecnología, capital, localización, etc., con frecuencia se cree que las EMN poseen mayor poder de negociación que los gobiernos nacionales.

Hay un gran debate sobre los posibles efectos de las EMN en el país huésped y más aún si éste se encuentra en un proceso de desarrollo. El análisis costobeneficio de dicha relación suele ser complicado y en cierta medida subjetivo y está fácilmente sometido a juicios de valor (Durán, 2001). No obstante, hay una amplia literatura (Sanna-Randaccio y Veugelers, 2003; Barrios et al., 2003; Feldstein, 2000; Gilpin, 2001; Bhagwati, 2004; Wolf, 2005; Sala-i-Martin, 2006) que coincide en que un flujo entrante de inversión proveniente de las EMN puede estimular el desarrollo local gracias al aumento y mejora de recursos y capacidades (stock de capital, tecnología, capacidad empresarial, acceso a mercados), aumento de competencia, mejor asignación de recursos, desarrollo de recursos humanos, generación de empleo, etcétera.

En este sentido, las empresas desearán desplazar sus recursos, como capital y tecnología, al extranjero cuando el rendimiento potencial es alto, sobre todo en los 
mercados en donde estos recursos son escasos. Ciertamente, la sola disponibilidad de recursos en un país no garantiza que éstos contribuirán a la producción; sin embargo, las EMN permiten usar esos recursos inactivos. Por ejemplo, la producción de petróleo requiere no sólo de la presencia de yacimientos petroleros, sino también del conocimiento de cómo encontrarlos, el equipo para extraerlo y las instalaciones para procesarlo. Simplemente extraer el petróleo es un desperdicio si no hay mercados ni instalaciones de transporte, los cuales puede proporcionar un inversionista extranjero.

La IED que realizan las EMN puede iniciar el mejoramiento de los recursos cuando entrena al personal local para operar nuevo equipo, en los métodos de producción y sobre todo para usar las nuevas tecnologías. La transferencia de métodos de trabajo innovadores aumenta la productividad, lo que a su vez incrementa el tiempo disponible para otras actividades. Además, la competencia adicional puede impulsar a las empresas existentes a mejorar su eficiencia.

Sin embargo, una de las críticas más extendidas en contra de las EMN es que éstas explotan a los trabajadores de los países en vías de desarrollo. De acuerdo con Bhagwati (2004: 259), "las empresas [multinacionales] que generan trabajo deberían ser aplaudidas, sin importar que su motivación para invertir en el extranjero sea obtener beneficios y no hacer el bien". Los críticos no coinciden con esta idea y basan sus argumentos en que las EMN pagan salarios más bajos en los países en vías de desarrollo. No obstante hay evidencia empírica y econométrica que demuestra lo contrario (Brown et al., 2002; Graham, 2000). Los estudios resaltan que el sueldo que pagan las EMN en los países en vías de desarrollo es superior a la media salarial del país. Por lo tanto, lo que hacen es pagar un salario competitivo, de acuerdo con las condiciones locales de cada país. Aunque con algún grado de cautela, la llegada de IED debe considerarse, en especial en los PMD, como una oportunidad de desarrollo y no como una amenaza.

Una crítica recurrente hacia las EMN es que son más poderosas que los propios gobiernos nacionales. Se dice que las más grandes del mundo tienen mayores presupuestos que algunos países en vías de desarrollo. Sin embargo, esta crítica carece de todo tipo de veracidad, ya que hay errores de interpretación por parte de los críticos que desvirtúan la realidad. En primer lugar, en el ámbito político, esas empresas no pueden compararse con la capacidad de coerción que tiene el gobierno sobre sus ciudadanos; en este sentido éste continúa teniendo el papel central. Por lo tanto, si las EMN se establecen bajo una jurisdicción, tendrán que desenvolverse conforme a las leyes competentes, que en última instancia las dicta el gobierno nacional. ${ }^{6} \mathrm{En}$ segundo lugar, la forma en la que los críticos establecen que las susodichas com-

${ }^{6}$ Sin embargo, para países muy pobres con industrias extractivas, las EMN sí han llegado en ocasiones a "imponer condiciones" a gobiernos débiles y/o corruptos (Stiglitz, 2006). 
pañías son económicamente más poderosas que algunos países, es comparando las ventas de éstas con el PIB de los países, las cuales son variables no comparables, ya que no miden lo mismo. Como consecuencia de esta crítica Grauwe y Camerman (2002) realizaron un estudio en el que comparan el valor agradado de las empresas y el resultado contradice radicalmente la crítica. Los autores concluyen que "las empresas son sorprendentemente más pequeñas comparadas con otros estados-nación" (Grauwe y Camerman, 2002: 15). Por lo tanto, los estados-nación continúan siendo agentes más importantes vis-à-vis las EMN.

No obstante, no todo es positivo en esas empresas; hay puntos ambiguos sobre sus actividades que tienen consecuencias perjudiciales, como son los daños que puedan ocasionar en el medio ambiente, la venta de productos dañinos, el soborno y corrupción que en ocasiones rodea a estas empresas. Aún más importante fueron los lobbies de las EMN para defender sus intereses ante la Organización Mundial de Comercio (OMC), lo que permitió que dicha institución pudiera aplicar sanciones a los países que infringieran el pago de regalías de la patente (Bhagwati, 2004: 276).

Problemas como estos deben abordarse de manera unilateral y multilateral, ya que sus consecuencias afectan a millones de personas en los países más pobres. Una de estas acciones tiene que comenzar desde dentro de los propios gobiernos nacionales, debido a que son sus leyes e instituciones las que permiten este tipo de abusos. De forma multilateral, la OMC deberá establecer reglas internacionales más claras y justas sobre las actividades de la EMN. Algunas otras acciones ya se han puesto en marcha, como la Ley de Prácticas Corruptas en el Extranjero, la cual es apoyada por un gran número de empresas estadounidenses, o los esfuerzos de innumerables organizaciones civiles nacionales e internacionales que, gracias a los avances tecnológicos, hacen posible que se conozca alrededor del mundo los diferentes abusos de las EMN.7

Pese a los posibles costos que pudiesen tener las EMN en el país de acogida, lo que se aprecia en las últimas dos décadas es que los PMD han aprendido que éstas, por medio de la IED, pueden ser de gran ayuda para su avance económico. Por ejemplo, durante el proceso de desarrollo en Brasil, el ex presidente Henrique Cardoso (1995-2002) $)^{8}$ realizó durante su mandato un gran esfuerzo para atraer a su país IED estadounidense (Gilpin, 2000). Del mismo modo en México, los diferentes presidentes en turno después de José López Portillo (1976-1982), observaron que una postura muy centralista o nacionalista daba como resultado una

\footnotetext{
${ }^{7}$ Véase Stiglitz (2006) para propuestas para disminuir los abusos de las EMN.

${ }^{8}$ Uno de los principales líderes de la teoría de la dependencia proponía en los años setenta que las EMN estadounidenses eran un instrumento del imperialismo estadounidense.
} 
disminución en la IED y por ende una posibilidad de desarrollo. Por tal motivo, las políticas de restricción a esa inversión han ido disminuyendo. De acuerdo con la UNCTAD (2007 y 2011), entre 1992 y 2010 se realizaron más de 2800 cambios regulatorios en las legislaciones nacionales sobre IED alrededor del mundo, siendo sólo el $10 \%$ de estos cambios menos favorables para la IED, ${ }^{9}$ mientras que el resto supuso una mayor liberalización.

Igualmente, los PMD han aprendido que las EMN son un vehículo de integración internacional, facilitando que un país participe en la división internacional del trabajo. Además, aquellos PMD que estén fuera de las redes globales de las EMN estarán en una desventaja considerable, principalmente porque parte del comercio internacional consiste en las transferencias intrafirma entre una filial y otra.

En conclusión, las EMN han tenido efectos positivos en los países de acogida, han creado empleos y en muchos casos contribuido al crecimiento económico de dichos países o regiones en donde se instalan. La mayoría de las críticas sobre la IED no tienen sentido cuando se analizan a fondo, aunque la distribución de los posibles beneficios que se tienen de las EMN en el país de acogida pueden ser asimétricos y por tanto son objeto de controversia. En este sentido, los abusos de esas empresas no se pueden dejar pasar por alto. Es aquí donde la sociedad civil y las leyes nacionales e internacionales desempeñan un papel importante para demandar y regular dichas acciones.

\section{LA ESTRATEGIA DE NEGOCIACIÓN DE LOS PMD FRENTE A LAS EMN}

Dentro de las negociaciones entre una EMN y un país de acogida, cada uno trata de obtener el máximo de concesiones sobre el otro. Podría pensarse que el país de acogida se encuentra en una ventaja relativa ( $\mathrm{vd} \mathrm{L}$ ), ya que puede controlar el acceso a su mercado, la mano de obra, y la materia prima necesaria y requerida para el buen funcionamiento de una EMN. Asimismo, si el país de acogida es grande, como el caso de la India, el mercado local también será una vd L que el país de acogida puede proporcionar a la EMN. Por ejemplo, de acuerdo con la India Brand Equity Fundation (2005), las multinacionales estadounidenses han hallado una gran ventaja en la India debido a su sistema democrático, al ambiente propicio para los negocios, pero fundamentalmente al tamaño del mercado local. Las EMN han encontrado un mercado donde se produce en gran cantidad, pero también

${ }^{9}$ Del conjunto de las regiones del mundo en 2010, la que realizó más cambios menos favorables para la inversión extranjera fue América Latina, reflejándose así el aumento en las regulaciones sobre la explotación de recursos naturales (UNCTAD, 2011). 
se consume enormemente. Con una población de 1000 millones de personas, el mercado local es sin duda una ventaja de L para la India.

Sin embargo, en la mayoría de los casos las ventajas de las EMN (ventajas de $\mathrm{o}$, [vd o]), como pueden ser tecnología, inversión, acceso a los mercados internacionales, etc., suelen ser de vital importancia para el desarrollo económico de un PMD, minimizando de inicio su posible poder de negociación. De esta manera, las vd L se ven superadas por las vd O, es decir, la EMN tendrá una ventaja sobre el país de acogida, el cual está falto de inversión. De acuerdo con Tarzi (2004), el poder económico de las EMN será aún mayor una vez que entra al mercado de PMD, en donde gran parte de la inversión interna estará en manos de la multinacional y ésta se concentrará en los sectores clave para el desarrollo del país de acogida. Por tal motivo, la concentración de la IED en sectores importantes de desarrollo, le dará a la EMN un poder monopolístico para controlar la oferta y los precios internos; cosa que no ocurriría en un mercado en donde la competitividad interna es mayor.

Por otro lado, Gilpin (2000) y Dunning (1993) sugieren que los PMD pueden obtener mayores beneficios de las EMN siempre y cuando el país de acogida modifique su estrategia de negociación. En este sentido, Tarzi (2004) propone que para que un PMD pueda influir en el comportamiento de una multinacional es importante observar y distinguir entre el poder actual y el potencial de un país de acogida. El poder actual de un PMD es la habilidad que tiene el gobierno del país de acogida para ejercer su poder de negociación a fin de obtener los mejores beneficios de la EMN. Este poder frecuentemente se ve limitado por diferentes acontecimientos que se presentan en escala global (presiones de instituciones internacionales o la situación económica mundial), así como por sucesos en el ámbito nacional (presión de ciertas clases sociales o la política interna), los cuales disminuyen las posibilidades de obtener mejores resultados en una negociación con una EMN.

A diferencia del poder actual, el potencial representa el poder relativo de negociación del gobierno de un PMD, el cual estará determinado por: a) la experiencia del gobierno local, b) el nivel de competitividad entre las empresas multinacionales, c) el grado de incertidumbre económica que exista, y d) el tipo de IED (Tarzi, 2004).

\section{EL NIVEL DE EXPERIENCIA DE LOS GOBIERNOS LOCALES}

La mayoría de los gobiernos en los PMD suelen contar con estructuras institucionales deficientes, lo cual se interpreta como un déficit institucional que pone a los gobiernos de acogida en una situación precaria para monitorear y gestionar a las EMN, es decir, algunos gobiernos de los PMD cuentan con poca experiencia para controlar las actividades de esas empresas. Una propuesta para solucionar este 
problema es que los países de acogida mejoren la calidad de sus instituciones gubernamentales a fin de vigilar de mejor manera a las multinacionales y aprovechar los beneficios que éstas les puedan proporcionar (Globerman y Shapiro, 2002). En esta propuesta no sólo se benefician las instituciones, pues la población trabajadora también va aplicando los nuevos conocimientos adquiridos por trabajar en la EMN, a la vez que se modernizan. Para poder obtener estos beneficios es preciso contar con una política abierta a la IED, ya que así el gobierno del país de acogida, por medio de la experiencia e interacción con las EMN, irá incorporando los nuevos conocimientos, a fin de mejorar y perfeccionar el control de las multinacionales. Sin embargo, si hubiese algún tipo de cambio en la tecnología, el cual va más rápido que las capacidades que tiene el país de acogida para absorberlo, éste se verá de nuevo en una desventaja frente a la EMN (Tarzi, 2004).

\section{EL NIVEL DE COMPETITIVIDAD ENTRE LAS EMPRESAS MULTINACIONALES}

La competencia o la falta de ésta entre las EMN puede afectar el poder de negociación del gobierno de un PMD. La inexistencia de competitividad entre aquéllas, supondrá una posición débil de negociación para el país de acogida, debido a que las multinacionales no reciben presión de las demás empresas por expandir y buscar nuevos nichos de mercado y de producción. En el caso contrario, el gobierno del PMD tendrá una mejor posición en el momento de la negociación; en este caso las multinacionales competirán por entrar al mercado del PMD y éste podrá seleccionar a la empresa que más beneficios aporte a la economía nacional. Del mismo modo, las ventajas que pueda ofrecer el PMD a las multinacionales (mano de obra barata, infraestructura desarrollada, materias primas), es muy importante para determinar cuál es la postura de negociación del país de acogida. Si la inversión que realizará la EMN es intensiva en capital o requiere de mano de obra cualificada y el PMD no cuenta con ella, la competencia de las multinacionales por entrar a ese mercado será limitada, alejando la posibilidad de negociación de dicho país (Tarzi, 2004). Conforme al paradigma de OLI, esto se interpreta de la siguiente manera: las EMN compiten por tener una ventaja en $\mathrm{O}$, y buscan un país de acogida que cuente con las mejores vd L, según sean las características de la inversión.

\section{INESTABILIDAD O INCERTIDUMBRE ECONÓMICA}

Las negociaciones entre las EMN y los gobiernos de los PMD siguen un patrón de comportamiento llamado "negociación obsoleta" (Tarzi, 2004; Gilpin, 2001). 
Este patrón consiste en que al comienzo de una negociación, el gobierno del PMD mantendrá una política de inversión favorable a fin de atraer a las EMN a sus mercados, lo que significa que éstas tendrán una ventaja al momento de la negociación, en donde querrán extraer el máximo de beneficios del PMD. Conforme pasa el tiempo y los proyectos invertidos empiezan a tener éxito, la ventaja inicial de las EMN comenzará a cambiar a favor del gobierno del país de acogida. En ese momento éste tendrá mejores ventajas que la EMN, la cual querrá renegociar los acuerdos iniciales (Tarzi, 2004). Sin embargo, el comportamiento de negociación obsoleta era más relevante cuando la IED se concentraba en sectores extractivos; ahora que la IED lo hace en el sector manufacturero y de servicios, ha perdido relevancia (Gilpin, 2001). Por ejemplo, una empresa que se dedica a la manufactura generalmente tiene mayor libertad de acción que aquellas que se dedican a la extracción de recursos naturales.

\section{EL TIPO DE INVERSIÓN EXTRANJERA DIRECTA}

El poder de negociación de un país de acogida dependerá en gran medida de las características de la IED. Éstas pueden ser: i) el volumen de la inversión física o fija; ii) el costo-beneficio; iii) el grado de complejidad tecnológica de la inversión, y iv) el grado de la complejidad de la mercadotecnia (Tarzi, 2004). De esta manera, las EMN analizarán y evaluarán las cuatro variables a fin de determinar la viabilidad de la inversión. Si los costos son bajos y la tecnología que se requiere es sencilla, el PMD tendrá una posición favorable en la negociación ya que cuenta con unas vd L propicias para ese tipo de inversión; de lo contrario estará fuera de las posibilidades de atraer a la EMN a su mercado.

De esta manera, se puede determinar que el poder potencial se construye de diversas posiciones en las cuales el gobierno de un PMD irá observando y analizando sus probabilidades de negociación frente a las EMN. Estas posiciones dependerán de lo que puedan ofrecer por un lado el país de acogida y por otro la multinacional, hasta llegar a un equilibrio de Nash.

Como se mencionó al comienzo de este apartado, el poder de negociación de los gobiernos de los PMD se ve afectado por diferentes circunstancias, es decir, por dificultades internas o por agentes externos al país de acogida. Dentro de las dificultades internas que limitan el poder de negociación de los PMD se encuentran, en primera instancia, sus instituciones y leyes deficientes, las cuales no permiten un óptimo manejo y control de las EMN. Del mismo modo, la falta de investigación y desarrollo ( $\mathrm{I}+\mathrm{D})$ debilita aún más su poder de negociación. También hay diferentes grupos sindicales y organizaciones sociales que presionan a 
los gobiernos para tomar decisiones forzadas respecto a la IED, como fue el caso de Bolivia durante 2003, cuando las uniones de campesinos indígenas, lideradas por Evo Morales (actual presidente de Bolivia), presionaron al gobierno para expropiar y limitar las actividades de las EMN en la extracción y comercialización del gas natural. Dichas manifestaciones fueron tan intensas que el presidente en turno, Gonzalo Sánchez de Lozada, tuvo que huir del país y presentar su dimisión.

Asimismo, la falta de competitividad de las empresas locales da una ventaja a las EMN para instalarse en los sectores importantes de desarrollo y monopolizarlos. La corrupción también es una debilidad que tienen los PMD. Hay evidencia (Egger y Winner, 2005; Daniels et al., 2004; Tarzi, 2004) de que la corrupción limita el poder de negociación del país de acogida. Los pagos a los funcionarios se realizan con la intención de asegurar un negocio a expensas de los competidores; facilitar los servicios gubernamentales que las empresas tienen derecho a recibir, pero que los funcionarios podrían retrasar, como el registro de productos, los permisos de construcción o las autorizaciones aduaneras; o simplemente garantizar la seguridad de los empleados e instalaciones. Igualmente, el cambio de gobierno en los PMD suelen ser objeto de un desequilibrio en el status quo entre los países de acogida y las EMN.

En lo referente a las dificultades internacionales que pueden limitar el poder de los gobiernos de los PMD se encuentran las redes globales creadas por las EMN. Éstas concentran gran parte del movimiento internacional de comercio y la mayoría de los PMD no tienen acceso a ellas. En general, las dificultades internacionales que puede tener el gobierno de un PMD dependerán de las necesidades que tengan las EMN de acuerdo con la tecnología, el tipo de mano de obra y las posibilidades del país de acogida para proporcionar dichas demandas. Igualmente, el país de origen de la EMN puede causar algunas limitaciones en el poder de negociación del PMD. El apoyo del país de origen se ve reflejado como un aspecto político. Por ejemplo, en el caso de Chile, en 1973, hay evidencia de que Estados Unidos apoyó el derrocamiento del presidente Allende con la finalidad de proteger los intereses de sus EMN en aquel país (Vidal, 2003).

En este sentido, la configuración del proceso de negociación entre el gobierno de un PMD y las EMN se basa en el concepto de costo de oportunidad. En el caso de las multinacionales viene dado por sus vd o, y las vd L ofrecidas por el país en el cual decidirá instalar su inversión. Para el caso del PMD, su costo de oportunidad vendrá dado por sus vd L y las vd o ofrecidas por las EMN. Por tanto, la multinacional estará en una mejor posición cuando sus costos de oportunidad son bajos y cuando el gobierno del PMD ponga un alto valor a la contribución económica y social de la EMN. En el caso contrario, el PMD se encontrará en una posición fuerte de negociación cuando 
sus recursos sean necesarios para la EMN y el análisis del costo de oportunidad esté a su favor, es decir, que pueda obtener los beneficios ofrecidos por la multinacional.

\section{CONCLUSIONES}

En este artículo se ha pretendido describir lo que es una empresa multinacional y los efectos que pueda tener sobre los países menos desarrollados; en especial se han descrito las capacidades y posibilidades de negociación que estos países tienen respecto de las multinacionales, a fin de obtener los mejores beneficios.

Como se describió en las secciones primera y tercera, la visión que tienen los gobiernos de PMD sobre las EMN ha ido cambiando a lo largo del tiempo. Durante principios y mediados del siglo XX, se veía a estas empresas como agentes internacionales de explotación y distorsionadores del mercado interno. No fue hasta finales de los años setenta y aun en la actualidad, cuando los PMD comenzaron a apreciar los beneficios de las multinacionales. Este cambio en la manera de verlas coincide con el aumento en la intensidad del proceso de globalización, el cual, por medio de la apertura comercial, ha permitido que las EMN se dispersen alrededor del mundo en mercados que anteriormente les estaban cerrados.

Sin lugar a dudas, los PMD, por sus propias características de atraso, son los que más dificultades tienen para atraer IED a sus economías, la cual, como se demostró con la evidencia empírica, puede tener efectos positivos para el desarrollo económico y servir de vínculo para incorporarse a las redes internacionales de transacciones y comercio. Del mismo modo, los gobiernos de los países menos desarrollados también son a los que se les complica más el proceso de negociación entre las multinacionales. En la sección cuarta se recrearon los diferentes panoramas en los que un gobierno de un PMD se podría encontrar en el momento de una negociación. En este sentido, es posible concluir que los PMD deben de contar con unas vd L, ${ }^{10}$ de tal modo que sean capaces de atraer la IED. Asimismo, el poder de negociación dependerá en gran medida de las habilidades que tenga cada uno de los que participan en la negociación. Por ejemplo, es importante que conozcan lo que le puede ofrecer el uno al otro. El resultado de la negociación dependerá también de la competencia y destreza del aparato burocrático del gobierno que esté llevando a cabo la operación.

${ }^{10}$ Ventajas del país de acogida, como incentivos fiscales, recursos naturales, infraestructura desarrollada, mano de obra calificada o no, etcétera. 
Sin embargo, el proceso de globalización ha modificado las necesidades de las EMN, por lo que en la actualidad las vd L de los PMD en muchas ocasiones no satisfacen las necesidades de las multinacionales, es decir, el costo de oportunidad de una EMN dedicada a la fabricación de software, el cual requiere de mano de obra calificada y tecnología de punta, será muy alto si decide invertir en un PMD, ya que éste no podrá proporcionar dichos bienes y habría que invertir en el desarrollo de esa tecnología. Por lo tanto, los PMD compiten por atraer IED, pero no cuentan con la infraestructura necesaria, lo que los aleja de la convergencia con los PD, convirtiéndose en un ciclo vicioso de pobreza.

En conclusión, los PMD deben mejorar sus vd L; para esto deberán crear sectores industriales competitivos, como lo sugiere el diamante de Porter, ${ }^{11}$ de tal manera que a las EMN les sea atractivo invertir en ese mercado. Así, los tomadores de decisiones en los PMD deberán crear políticas públicas enfocadas a mejorar sus ventajas competitivas restructurando prácticamente todo su sistema productivo, institucional y social, dando prioridad a la educación, la igualdad de género, la seguridad jurídica, y a los incentivos, a fin de propiciar el flujo de IED, además de encontrar aquel nicho en el cual son competitivos. El escenario para los PMD no está del todo claro, ya que cada vez es más difícil atraer IED a sus mercados debido a que en los últimos años esas empresas tienden a instalarse principalmente en los países desarrollados y aquellos catalogados como emergentes, debido a que éstos pueden ofrecer mejores vd L que los PMD, y, sobre todo, han cambiado las vd o de las multinacionales, modificando la geografía de las IED (Dunning, 2000). Asimismo, es preciso mencionar que se debe contar con un agente supranacional, como la OMC u otra organización internacional, que se encargue de la regulación de las IED y las EMN con la finalidad de intervenir a favor de los países de acogida y dar así una esperanza a los menos desarrollados de enfrentar de mejor manera el proceso de globalización; sin embargo, este es un tema para una investigación más profunda.

\section{BIBLIOGRAFÍA}

Anderson, Sara y John Cavanagh (2000), Top 200, the rise of corporate global power, Institute for Policy Studies, diciembre.

${ }^{11}$ El diamante de Porter muestra la interacción de cuatro condiciones, las cuales generalmente deber ser favorables para que una industria de un país pueda obtener una ventaja competitiva global, y éstas son: demanda, dotación de factores, industrias afines y de apoyo, y estrategia, estructura y rivalidad entre las empresas. La forma en que se combinen estas condiciones influye en el avance y fortaleza de las ventajas competitivas (Porter, 1990). 
Barrios, Salvador et al. (2003), "Foreign direct investment, competition and industrial development in the host country", European Economic Review, núm. 49, pp. 1761-1784.

Bhagwati, Jagdish (2004), In defence of globalization, Oxford University Press. Brown, Drusilla et al. (2002), "The effects of multinational production on wages and working conditions in developing countries", Research Seminar in International Economics, Discussion Paper núm. 484, agosto, Universidad de Michigan.

Daniels, John D. et al. (2004), Negocios internacionales, ambientes y operaciones, 10a ed., México, Pearson Educación.

De la Dehesa, Guillermo (2004), Comprender la globalización, Madrid, Economía Alianza Editorial.

Dunning, John H. (1993), Multinational enterprises and the global economy, Inglaterra, Addison-Wesley.

(1999), "Globalization and the theory of MNE activity", Discussion Papers en International Investment and Multinationals, Series B, vol. XI (1998/1999), núm. 264, University of Reading, Department of Economics.

(2000), "Globalization and the new geography of foreign direct investment", en Ngaire Woods, The political economy of globalization, Londres, Palgrave.

Dunning, John H., y Narula Rajneesh (1997), "Developing countries versus multinationals in a globalising world: dangers of falling behind", Discussion Papers en International Investment and Multinationals, Series B, vol. IX (1996/1997), núm. 226, University of Reading, Department of Economics.

Durán, Juan José (2001), Estrategia y economía de las empresas multinacionales, España, Pirámide.

Egger, Peter, y Winner Hannes (2005), "Evidence on corruption as an incentive for foreign direct investment", European Journal of Political Economy, vol. 21, pp. 932-952.

Evans, Graham, y Newnham Jeffrey (1998), The Penguin dictionary of international relations, Inglaterra, Penguin Books.

Feldstein, Martin (2000), "Aspects of global economic integration: outlook for the future", NBER, Working Paper, núm. 7899.

Gilpin, Robert (2000), The challenge of global capitalism, the world economy in the $21^{\text {st }}$ century, Princeton, Princeton University Press.

Gilpin, Robert (2001), Global political economy: understanding the international Economic order, Princeton University Press.

Globerman, Steven y Daniel Shapiro (2002), "Global foreign direct investment flows: the role of governance infrastructure", World Development, vol. 30, núm. 11, pp. 1899-1919. 
104 ECONOMÍA: TEORÍA Y PRÁCTICA • NUEVA ÉPOCA, NÚMERO 36, ENERO-JUNIO 2012

Goldberg, Linda (2004), "Financial-sector FDI and host countries: new and old lessons", NBER, Working Paper 10441.

Graham, Edward (2000), Fighting the wrong enemy antiglobal activists and multinational enterprises, Institute for International Economics, Washington, D.C.

Grauwe, Paul, y Filip Camerman (2002), How big are the big multinationals companies?, Universidad de Leuven y Senado Belga. En http://www.econ. kuleuven.be/ew/academic/intecon/Degrauwe/PDG-papers/Recently published articles/How\%20big\%20are\%20the\%20big\%20multinational\%20companies.pdf, Consultada en julio de 2010.

Hirts, Paul, y Thompson Grahame (1996), Globalization in question, $2^{\mathrm{a}}$ ed., Polity Press, Cambridge, Reino Unido.

Hymer, Stephen H. (1976), The international operation of national firms: a study of foreign direct investment, MIT Press.

India Brand Equity Fundation (2005), "It is destination India for US companies", IBEF Study.

Instituto Mexicano del Petróleo, http://www.imp.mx/. Consultada en mayo de 2010.

Krugman, Paul R. y Obstfeld Maurice (1999), Economía internacional, teoría y política, $4^{\mathrm{a}}$ ed., México, Mc Graw Hill.

Lipsey, Robert E. (2001), "Foreign direct investment and the operations of multinationals firms: concepts, history and data", NBER, Working Paper 8665.

Porter, Michael E. (1990), The Competitive Advantage of Nations, Londres, The Macmillan Press.

Sala-i-Martin, Xavier (2006), Globalización y reducción de la pobreza, FAES, España.

Sanna-Randaccio, y Veurgeles Reinhidle (2003), "Global innovation strategies of MNE's: implications for host economies", en John Cantwell y José Molero, Mutinational enterprises, innovative strategies and systems of innovation, Cheltenham, Edward Elgar Publishing.

Stiglitz, Joseph (2006), Cómo hacer que funcione la globalización, Taurus, Madrid.

Tarzi, Shah M. (2004), "Third world governments and multinational corporations: dynamics of host's bargaining power", en Jeffry A. Frieden y David Lake, International political economy, perspective on global power and wealth, 4a ed., Londres, Routledge.

Taylor, Alan M. (1999), "Latin America and foreign capital in the twentieth century: economics, politics, and institutional change", NBER, Working Paper 7394. 
UNCTAD (2007), World investment report 2007, transnational corporations, extractive industries and development, ONU, Nueva York y Ginebra.

UNCTAD (2008), Los países menos adelantados. Informe de 2008, ONU, Nueva York y Ginebra.

- (2008a), World investment report 2008, transnational corporations and the infrastructure challenge, ONU, Nueva York y Ginebra.

- (2011), World investment report 2011, non equity modes of international production and development, ONU, Nueva York y Ginebra.

Vernon, Raymond (1973), Soberanía en peligro. La difusión multinacional de las empresas de Estados Unidos, México, Fondo de Cultura Económica.

Vidal, César (2003), “¿Por qué tuvo lugar el golpe de Estado de 1973 en Chile?”, La Ilustración Liberal, Revista española y americana, núm. 18.

Willetts, Peter (2001), "Transnational actors and international organizations in global politics", en John Baylis y Steve Smith, The globalization of world politics, an introduction to international relations, 2a ed., Reino Unido, Oxford University Press.

Wolf, Martin (2005), Why globalization works, Yale Nota Bene, Reino Unido. 